\title{
Planck on Extragalactic Radio Sources: Data and Findings
}

\author{
Bruce Partridge $^{1}$, on behalf of the Planck Collaboration ${ }^{2}$ \\ ${ }^{1}$ Haverford College Astronomy Department, 370 Lancaster Avenue, Haverford, Pennsylvania, \\ U.S.A. \\ email: bpartrid@haverford.edu \\ ${ }^{2}$ http:// www.cosmos.esa.int/web/planck/planck-collaboration
}

\begin{abstract}
Planck scanned the entire sky every six months at nine frequencies bands from 28 to $857 \mathrm{GHz}$ with enough sensitivity to detect over a thousand radio sources. It thus provided measurements of the $\mathrm{mm}$ and sub-mm spectra of these sources in a regular cadence, even at wavelengths hard to observe from the ground. Polarization measurements (or upper limits) are provided for brighter sources at $28-353 \mathrm{GHz}$. Finally, Planck is calibrated to $<1 \%$ accuracy in most of its frequency bands. I briefly introduce the valuable data set Planck provides on extragalactic sources, then describe some of the scientific conclusions drawn from the Planck measurements.
\end{abstract}

A radio astronomer's dream survey might have the following features: it would cover the entire sky; it would be conducted at many frequencies (including those difficult to reach from the ground); it would provide full Stokes polarization measurements; it would be repeated with several cadences; and it would be absolutely calibrated. This is precisely what the Planck mission has provided: all-sky surveys and catalogues of sources at nine frequencies, $\sim 28,44,70,100,143,217,353,545$ and $857 \mathrm{GHz}$, made over at least five independent surveys (eight surveys at the 3 lowest frequencies).

Planck has detected in its 5-6 lowest frequency bands the $\sim 1000$ brightest radio sources, most of them extragalactic. It has also detected an even larger number of FIR sources, discussed by López-Caniego later in this volume. Planck's measurements of compact sources are contained in the nine catalogues making up the PCCS2, one for each Planck frequency (see the detailed report by López-Caniego here).

I now briefly describe some results on extragalactic radio sources provided by the Planck surveys, and give references to the Planck papers that present detailed results. Perhaps the most fundamental result of the surveys is that Planck detected no new category of sources. Instead, it permitted refined counts of sources (Planck Collab. XXVI, 2015; see Fig. 1) and luminosity functions (Negrello et al., 2013). Even early Planck results showed that source counts at all frequencies up to and including $217 \mathrm{GHz}$ were dominated by flat-spectrum radio sources (blazars and other AGN; Planck Intermed. Results VII, 2013).

Planck also provided microwave spectra for hundreds of sources, including measurements at frequencies hard to reach from the ground. Fig. 2 shows an example, a source with both synchrotron emission and re-emission from hot dust (Lähteenmäki et al. 2015). At $28-70 \mathrm{GHz}$, there are 8 full-sky surveys, each lasting six months (and 5 surveys at $100 \mathrm{GHz}$ and above). The repeated cadence has proven very useful for studies of radio source variability (see Chen et al. 2013 and Lähteenmäki et al. 2015).

Finally, Planck is absolutely calibrated to better than $1 \%$ precision at the frequencies of interest here using the CMB dipole induced by the satellite's yearly motion around 


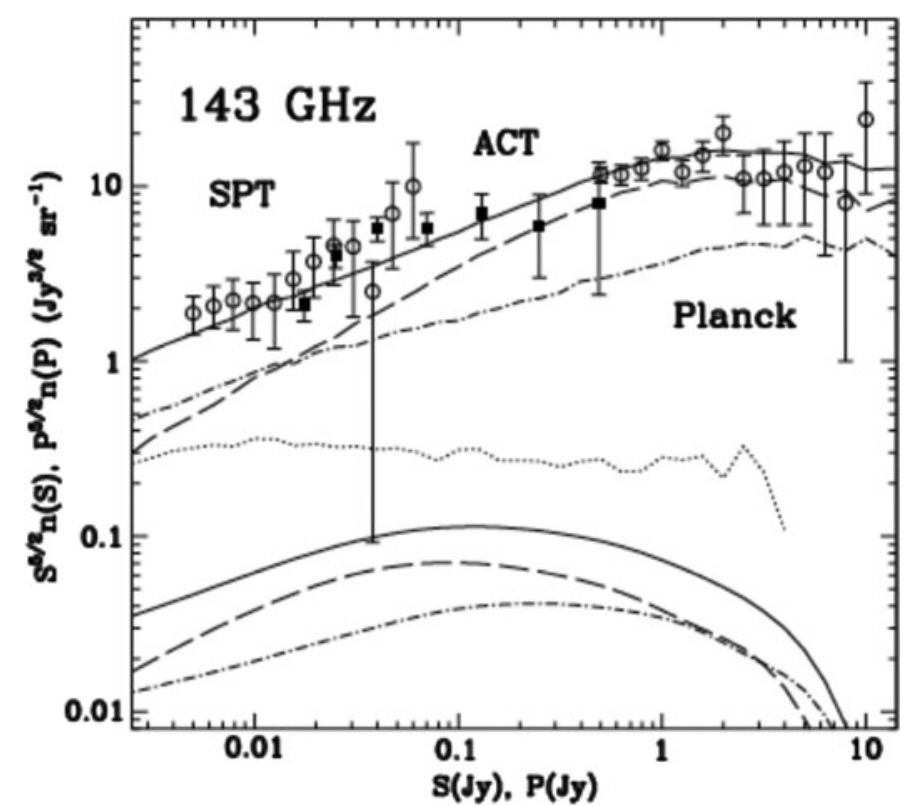

Figure 1. Source counts at $143 \mathrm{GHz}$; Planck supplies the counts at the bright end; the upper lines are models of source counts by Tucci et al. 2011, which fit the counts of Radio sources well. Their predictions of the counts of polarized sources are shown by the three lower curves.

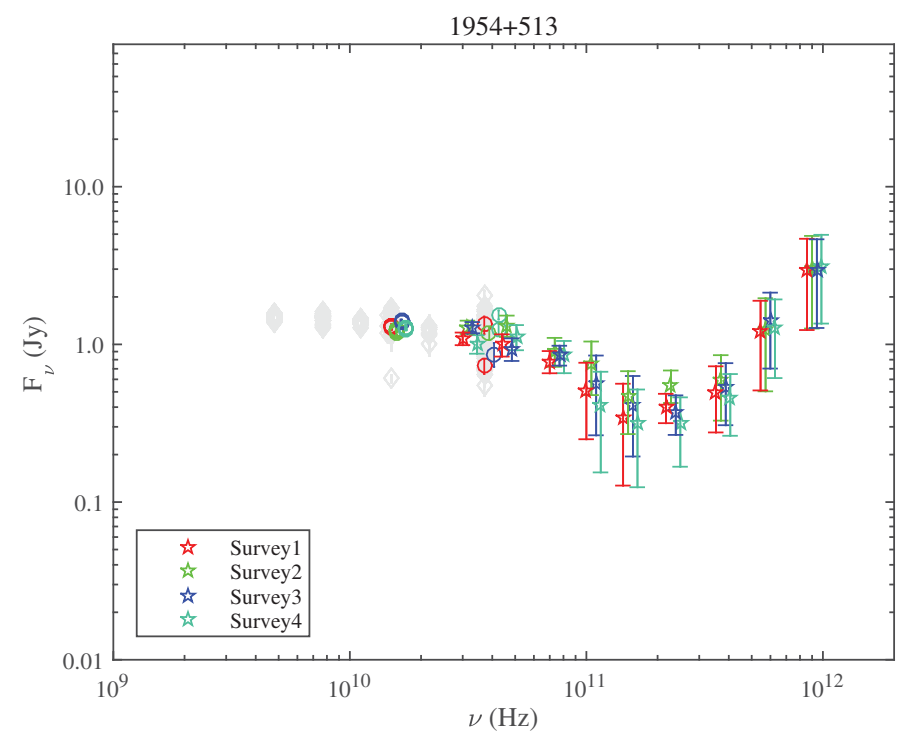

Figure 2. A sample Planck SED; the agreement from survey to survey show that this source does not measurably vary.

the Sun (Planck Collab. I 2015). This calibration has now been extended to ground based radio telescopes by direct comparison of Planck observations of sources and nearly simultaneous ground-based observations of the same sources (for the VLA and ATCA, see Partridge et al. 2015; for ACT, Louis et al. 2014). One example is shown in Fig. 3.

Much more can be done with Planck observations; we welcome wider use of Planck maps and catalogues by the radio astronomical community. 


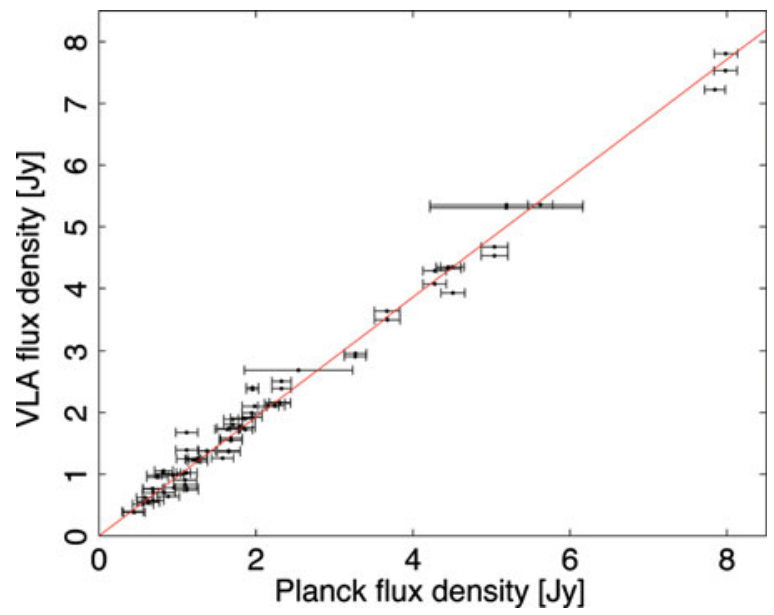

Figure 3. Comparison between Planck and VLA measurements at $28.45 \mathrm{GHz}$; scatter arises from variability. Slope $=0.964 \pm 0.008$; the VLA values run $\sim 3.6 \%$ below Planck's, within the VLA uncertainty of $\pm 5 \%$.

\section{References}

Chen et al. 2013, A\&BA, 553, 107C

Lähteenmäki et al. 2015 , in press at $A \mathscr{E} A$

Louis et al. 2014, JCAP 7, 016

Negrello et al. 2013, MNRAS 429, 1309

Partridge et al. 2015, in press at $A p J$

Planck Collab. I 2015, Planck Collab. XXVI, 2015, in press at $A \mathscr{G} A$; arXiv:1502.01582 and arXiv:1507.02058

Planck Intermediate Results VII 2013, in press at $A \& A$

Tucci, et al. 2011, A\&SA 533, A57 